\title{
IAEA compiling state of knowledge and developing high level guidance in radioactive waste management
}

\author{
Stefan Mayer and Rebecca Robbins \\ IAEA, Vienna, Austria \\ Correspondence: Stefan Mayer (s.j.mayer@iaea.org) \\ Published: 10 November 2021
}

\begin{abstract}
One endeavour central to the International Atomic Energy Agency's (IAEA) mandate is compiling the international state of knowledge (SoK), supporting transfer of knowledge and establishing high level guidance for all peaceful applications of nuclear technologies, including those specific to radioactive waste management (RWM).

Building on the current state of experiences and knowledge shared with us from IAEA Member States, we are developing a comprehensive suite of documents on the technologies and solutions having the potential to address RWM needs and steps. This information can be used as a baseline to guide RWM in other countries.

In a first approach, the information is compiled and developed in a sequence of documents covering a range of topical domains. These include:
\end{abstract}

1. RW: inventory; characterization; use of waste acceptance criteria throughout all RWM steps.

2. RWM steps: a series of handbooks presenting approaches to waste treatment, conditioning and storage; special applications supporting small inventory management needs (e.g. through mobile facilities); considerations specific to management needs of postaccident radioactive waste.

3. Endpoints: introduction to design approaches and disposal concepts; managing site investigations; global compendium of Underground Research Facilities (URF) RD\&D; special considerations for small inventory disposal.

4. Programmatic considerations: a generic roadmap to deep geologic repository (DGR) implementation; cost estimation and funding of a disposal programme; stakeholder involvement in disposal.

To improve on efficiency and to prove our capacity to compile and transfer an evolving state of knowledge on topics relevant to RWM, the IAEA is building a collection of online interactive contents based on these publications. This online interactive content ultimately aims at containing all our key guidance topics and is intended to provide easier and more rapid access to detailed information. The format would also allow its content to be expanded with descriptions and analyses of topical case studies.

Kurzfassung. Ein zentrales Unterfangen des Auftrags der Internationalen Atomenergie-Organisation (IAEA) besteht darin, den internationalen Wissensstand zu erfassen und so den Wissenstransfer und die Etablierung von Orientierungshilfen auf hohem Niveau für sämtliche friedlichen Anwendungen nuklearer Technologien zu unterstützen, einschließlich der Technologien, die spezifisch der Entsorgung radioaktiver Abfälle dienen.

Auf der Grundlage des aktuellen Erfahrungs- und Wissensstands im Austausch mit den IAEAMitgliedsstaaten entwickelt die IAEA eine umfangreiche Reihe von Dokumenten zu den Technologien und Lösungen, die das Potenzial besitzen, Erfordernisse und Schritte hinsichtlich der Entsorgung radioaktiver Abfälle anzugehen. Diese Informationen können als Ausgangspunkt für die Anleitung zur Entsorgung radioaktiver Abfälle in anderen Ländern verwendet werden.

In einem ersten Ansatz werden Informationen in einer Folge von Dokumenten mit einer Reihe von Themengebieten erfasst und nutzbar gemacht. Dazu gehören: 
1. Radioaktive Abfälle: Inventar; Charakterisierung; Anwendung der Kriterien für die Annahme von Abfällen während sämtlicher Schritte zur Entsorgung radioaktiver Abfälle

2. Schritte zur Entsorgung radioaktiver Abfälle: eine Reihe von Handbüchern mit Ansätzen zur Abfallbehandlung, -konditionierung und -lagerung; spezielle Anwendungen zur Unterstützung der Erfordernisse kleiner Bestandsverwaltung (z. B. in Form mobiler Anlagen); spezifische Überlegungen in Bezug auf die Erfordernisse für den Umgang mit radioaktiven Abfällen nach Zwischenfällen

3. Endpunkte: Einführung in Gestaltungsansätze und Entsorgungskonzepte; Leitung von Standortuntersuchungen; globales Kompendium zu Forschung, Entwicklung und Demonstration (RD\&D) bei unterirdischen Forschungsanlagen (URF); spezielle Überlegungen für die Entsorgung kleiner Bestände

4. Programmatische Überlegungen: allgemeiner Fahrplan zur Implementierung geologischer Tiefenlager (DGR); Kostenabschätzung und Finanzierung eines Entsorgungsprogramms; Einbeziehung der Beteiligten und Entsorgung

Für eine bessere Effizienz und für den Nachweis der Fähigkeit der IAEA, den wachsenden Wissensstand zu Themen mit Relevanz für die Entsorgung radioaktiver Abfälle zu erfassen und weiterzuvermitteln, baut die IAEA eine Sammlung von interaktiven Online-Inhalten auf der Grundlage dieser Publikationen auf. Ziel dieser interaktiven Online-Inhalte ist es letztendlich, alle wichtigen Leitlinienthemen zu enthalten sowie leichteren und schnelleren Zugang zu detaillierten Informationen zu ermöglichen. Das Format erlaubt auch die Erweiterung des Inhalts um Beschreibungen und Auswertungen aktueller Fallstudien. 